\title{
Effect of the distribution of faults and operating conditions on AFDD performance evaluations
}

\author{
David P. Yuill $^{\mathrm{a},{ }^{*}}$ and James E. Braun ${ }^{\mathrm{b}}$
}

${ }^{a}$ Durham School of Architectural Engineering and Construction, University of Nebraska Lincoln, 1110 S. 67th Street, Omaha, NE 68182, USA.

${ }^{\mathrm{b}}$ School of Mechanical Engineering, Purdue University, 585 Purdue Mall, West Lafayette, IN 47907, USA.

* Corresponding author: +1-402-554-4176, dyuill@unl.edu

\begin{abstract}
Automated fault detection and diagnosis (AFDD) tools are used to identify degradation faults that reduce the performance and life of air-conditioning equipment. A recent methodology has been developed to evaluate the performance of AFDD tools. The methodology involves feeding a library of input data to an AFDD protocol and categorizing the results. The current paper describes a study that has been conducted to assess the effect of using various input data sets in the evaluations. These input data sets include different distributions of fault type, fault intensity, and operating temperatures. Case study evaluations of three AFDD protocols in current widespread use are used to demonstrate the effects. The paper shows that evaluation results are sensitive to input data sets, and argues that data sets used in previously published studies should be improved to give higher fidelity evaluations. It concludes that for AFDD performance evaluation to be meaningful, the fault and operating conditions need to be controlled so that they connect to the anticipated deployment conditions. A related conclusion is that it is necessary to use simulation data, rather than laboratory measurement data, to conduct performance evaluation of AFDD.
\end{abstract}

Keywords: AFDD, FDD, fault, detection, diagnosis, evaluation

\section{Nomenclature}

$\begin{array}{ll}\text { AC } & \text { Air conditioner } \\ \text { AFDD } & \text { Automated fault detection and diagnosis } \\ \text { CA } & \text { High side heat transfer fault } \\ \text { Delta } & \text { Difference } \\ \text { EA } & \text { Low side heat transfer fault } \\ \text { FI } & \text { Fault Intensity } \\ \text { FIR } & \text { Fault Impact Ratio } \\ \text { FXO } & \text { Fixed orifice expansion device } \\ \text { HP } & \text { Heat pump } \\ \text { LL } & \text { Liquid line restriction fault } \\ \text { m } & \text { mass } \\ \dot{m} & \text { mass flow rate }\end{array}$




$\begin{array}{ll}\text { NC } & \text { Non-condensable gas in refrigerant fault } \\ \text { NoF } & \text { No fault } \\ \text { OC } & \text { Overcharge of refrigerant fault } \\ \text { P } & \text { Pressure } \\ \text { Q } & \text { Air-conditioner capacity } \\ \text { T } & \text { Temperature } \\ \text { TXV } & \text { Thermostatic expansion valve } \\ \text { UC } & \text { Undercharge of refrigerant fault } \\ \dot{V} & \text { Volumetric flow rate } \\ \text { VL } & \text { Compressor fault } \\ \text { WB } & \text { Thermodynamic wet bulb temperature }\end{array}$

$\begin{array}{ll}\text { Subscripts } \\ \text { amb } & \text { Ambient } \\ \text { cop } & \text { Coefficient of performance } \\ \mathrm{N}_{2} & \text { Nitrogen gas } \\ \text { ra } & \text { Return air } \\ \text { ref } & \text { Reference }\end{array}$

\subsection{Introduction}

Automated Fault Detection and Diagnosis (AFDD) tools have the potential to detect performance degradation faults early so that the faults can be addressed before they would otherwise be detected [1]. Addressing faults can increase equipment life, improve performance and efficiency, and avoid unscheduled loss of service. AFDD tools also provide a diagnosis of the type or location of the fault, and in some tools, an assessment of the fault's severity or impact [2].

AFDD tools are used in many heating, ventilation, and air-conditioning (HVAC) applications, and their usage is becoming more widespread [3-8]. An important question that naturally arises is this: how well do these tools perform? Yuill and Braun [9-10] developed a methodology to evaluate the performance of the protocols used in handheld AFDD tools that are applied to unitary air-cooled equipment, such as rooftop units (RTU) and split systems. The methodology is briefly summarized below, and involves feeding input data for a given scenario into the AFDD protocol, and comparing the protocol's response to the known fault condition. The scenario includes the specific piece of unitary equipment that the AFDD might be applied to, the operating conditions, and the fault condition.

To characterize AFDD performance over a wide range of operating conditions and potential fault conditions, the process is repeated over a large number of scenarios, and the results are organized according to: (a) the outcome; and (b) the impact of the fault on system capacity or coefficient of performance (COP). Statistics are generated to summarize overall performance.

A taxonomy for the six potential outcomes of each scenario is proposed: 1. No Response; 2. Correct; 3. False Alarm; 4. Misdiagnosis; 5. Missed Detection; 6. No Diagnosis. The fault's performance impact is quantified for each scenario using Fault Impact Ratio (FIR), 
which is the ratio of performance with the fault, to performance under the same conditions if the fault were not present, and is defined for efficiency (COP) and capacity (Q): $F I R_{C O P}$ $=C O P_{\text {faulted }} / C O P_{\text {unfaulted }}$ and $F I R_{Q}=Q_{\text {faulted }} / Q_{\text {unfaulted }}$.

Applying the methodology to a specific evaluation method requires selecting an appropriate set of inputs to be fed to the protocol. [10] presents a case study in which they use laboratory measurement data gathered from multiple experimenters and laboratories (all reliable data that they could gain access to) for the evaluation. The case study shows the outcome from evaluating a widely-used AFDD protocol. In the paper, the faults that are considered are shown in Table 1, along with the laboratory implementation of the fault.

Table 1: Fault types and laboratory implementation in input libraries

\begin{tabular}{|l|l|l|}
\hline Fault & Abbr. & Laboratory Implementation \\
\hline Undercharge & UC & Refrigerant is removed \\
\hline Overcharge & OC & Refrigerant is added \\
\hline High-side heat transfer fault & CA & Airflow across condenser is reduced \\
\hline Low-side heat transfer fault & EA & Airflow across the evaporator is reduced \\
\hline Non-Condensable & NC & Nitrogen gas is added to the refrigerant \\
\hline Liquid Line Restriction & LL & A valve is inserted in the liquid line \\
\hline Compressor leakage & VL & Hot gas is bypassed to the compressor suction \\
\hline
\end{tabular}

Cheung and Braun [11] developed a gray-box modeling method that is capable of modeling fault effects on all of the system parameters typically used by AFDD tools. They used the approach to model eight of the systems that are included in Yuill and Braun's [10] measurement data library.

A major drawback to using measurement data to evaluate AFDD tools is that they are costly to generate, because they require a significant amount of laboratory time and highly skilled technicians. To reasonably characterize overall performance, a large number of scenarios is needed - several hundred - since AFDD tools perform better at some operating and fault conditions than others. Yuill and Braun's [11] approach of using preexisting data has the drawback that they couldn't control the distribution of input conditions. The data library is dominated by certain fault types (refrigerant undercharge, for example) and certain operating conditions $\left(95^{\circ} \mathrm{F}\right.$ outdoor drybulb, $80^{\circ} \mathrm{F}$ indoor drybulb, and $67^{\circ} \mathrm{F}$ indoor wetbulb $\left(35 / 27 / 19^{\circ} \mathrm{C}\right)$, for example). Using the library of existing data that contains these scenarios will skew the results in favor of protocols that are more effective with these fault types and operating conditions.

With Cheung and Braun's [11-12] models available, evaluations can be made using inputs at a controlled set of conditions [13]. However, a major drawback to using simulations is that engineers and scientists not involved with a model's development are often skeptical of the model's outputs. To address this, a validation of the models was conducted [14]. But even with valid models to use, the question arises: what distribution of input scenarios (fault conditions and operating conditions) should be used? An even distribution of conditions may provide a good characterization of overall performance, but it doesn't necessarily connect to how well the AFDD tool will perform when deployed in the field. For example, if a slightly fouled condenser is believed to be more likely than a severely 
fouled condenser, we may be more concerned with this fault condition. The same holds true of operating conditions.

\subsection{Method}

To study the issues involved with using measurement data and model data the current paper shows the results of evaluating three AFDD protocols, each evaluated with five sets of input data. The protocols are currently used commercial and public AFDD tools. In general, AFDD tools detect and diagnose faults by comparison of measurements with expected values, which typically come from analytical redundancy, such as models, or from heuristic rules [15-17]. Common input variables for unitary air-conditioners include suction superheat, liquid-line subcooling, and air-side temperature change across the evaporator or condenser, but there is a wide variety of AFDD approaches that use other inputs in addition to these [18]. For example, Armstrong et al. [19] use only high frequency electrical power measurements for fault detection.

The input data sets are as follows:

A. A library of laboratory-gathered measurement data from 8 air-conditioners

B. Modeled data that exactly simulate the laboratory measurements of set A. i.e. models of the same air-conditioners, operating conditions, and fault conditions as the laboratory data.

C. Modeled data from the same eight air-conditioners as sets A and B, but at a set of operating conditions and fault conditions that is uniformly distributed in the input space.

D. Modeled data from set $\mathrm{C}$, but weighted according to the probability of each condition arising during field deployment of the AFDD tool.

\subsection{Inputs}

All four sets of inputs represent the operation of 8 different air conditioning systems, which are described in Table 2. This table also shows the number of fault tests that were conducted in laboratory measurements for each system for each fault type. There is a total of 587 tests that comprise input data set A.

Table 2: Description of test units for all input sets, and operating conditions in data sets A and B

\begin{tabular}{|c|c|c|c|c|c|c|c|c|c|c|c|c|c|c|}
\hline \multirow[b]{2}{*}{ Config. } & \multirow[b]{2}{*}{ Type } & \multirow[b]{2}{*}{$\begin{array}{c}\text { Capacity } \\
{[k W]}\end{array}$} & \multirow[b]{2}{*}{ Refrig. } & \multirow[b]{2}{*}{$\begin{array}{c}\text { Exp. } \\
\text { Device }\end{array}$} & \multicolumn{8}{|c|}{ Number of tests } & \multicolumn{2}{|c|}{$\begin{array}{l}\text { Ambient } \\
\text { Temp. }\end{array}$} \\
\hline & & & & & $\begin{array}{c}\text { No } \\
\text { Fault }\end{array}$ & UC & OC & EA & CA & LL & NC & VL & $\begin{array}{l}\text { Min. } \\
{\left[{ }^{\circ} \mathrm{C}\right]}\end{array}$ & $\begin{array}{l}\text { Max. } \\
{\left[{ }^{\circ} \mathrm{C}\right]}\end{array}$ \\
\hline RTU & $A C$ & 10.5 & R410A & FXO & 24 & 25 & 12 & 21 & 6 & 0 & 0 & 0 & 19 & 52 \\
\hline RTU & $A C$ & 10.5 & $\mathrm{R} 22$ & FXO & 39 & 34 & 0 & 26 & 36 & 34 & 0 & 33 & 16 & 38 \\
\hline RTU & $A C$ & 17.6 & R407C & FXO & 17 & 15 & 12 & 19 & 8 & 0 & 0 & 0 & 19 & 47 \\
\hline Split & $A C$ & 10.5 & R410A & FXO & 1 & 29 & 1 & 0 & 0 & 0 & 0 & 0 & 28 & 53 \\
\hline Split & $\mathrm{HP}$ & 8.8 & R410A & TXV & 16 & 12 & 12 & 21 & 15 & 16 & 15 & 16 & 21 & 38 \\
\hline Split & $A C$ & 10.5 & R410A & TXV & 2 & 30 & 7 & 0 & 0 & 0 & 0 & 0 & 28 & 53 \\
\hline Split & $\mathrm{HP}$ & 10.5 & R410A & TXV & 4 & 4 & 7 & 0 & 0 & 0 & 0 & 0 & 28 & 52 \\
\hline Split & $\mathrm{HP}$ & 10.5 & $\mathrm{R} 22$ & TXV & 4 & 4 & 4 & 6 & 0 & 0 & 0 & 0 & 28 & 52 \\
\hline & & & & Total: & 107 & 153 & 55 & 93 & 65 & 50 & 15 & 49 & & \\
\hline
\end{tabular}


Input data set $\mathrm{C}$ has a uniform grid of conditions for each of the 8 air conditioners. The grid contains all physically possible combinations of the operating conditions listed in Table 3, totaling 48 .

Table 3: Operating conditions for input data set C

\begin{tabular}{|c|c|c|}
\hline & {$\left[{ }^{\circ} \mathrm{C}\right]$} & {$\left[{ }^{\circ} \mathrm{F}\right]$} \\
\hline $\mathrm{T}_{\mathrm{ra}}$ & 21.125 .028 .9 & 707784 \\
\hline$W B_{r a}$ & 12.818 .323 .9 & $55 \quad 6575$ \\
\hline $\mathrm{T}_{\mathrm{amb}}$ & $18.3 \quad 23.9 \quad 29.4 \quad 35.0 \quad 40.646 .1$ & $65 \quad 75 \quad 8595 \quad 105 \quad 115$ \\
\hline
\end{tabular}

There are 32 single-fault conditions (multiple simultaneous faults are not considered in Yuill and Braun's [10] methodology). These fault conditions include a fault type (e.g. liquid line restriction) and a Fault Intensity (FI). FI is a quantifier of the severity of the fault with respect to directly measurable quantities. It is defined for each fault type in [10], and summarized in Table 4.

Table 4: Fault intensity definitions

\begin{tabular}{|l|l|}
\hline Fault type & Fault Intensity Definition \\
\hline $\begin{array}{l}\text { UC or } \\
\text { OC }\end{array}$ & $F I_{\text {charge }}=\frac{m_{\text {actual }}-m_{\text {nominal }}}{m_{\text {nominal }}}$ \\
\hline EA & $F I_{E A}=\frac{\dot{V}_{\text {actual }}-\dot{V}_{\text {nominal }}}{\dot{V}_{\text {nominal }}}$ \\
\hline CA & $F I_{C A}=\frac{\dot{V}_{\text {actual }}-\dot{V}_{\text {nominal }}}{\dot{V}_{\text {nominal }}}$ \\
\hline LL & $F I_{L L}=\frac{\Delta P_{L L, \text { faulted }}-\Delta P_{L L, \text { unfaulted }}}{\Delta P_{L L, \text { unfaulted }}}$ \\
\hline $\mathrm{NC}$ & $F I_{N C}=\frac{m_{N_{2}, \text { faulted }}}{m_{N_{2}, \text { ref }}}$ \\
\hline VL & $F I_{V L}=\frac{\dot{m}_{\text {faulted }}-\dot{m}_{\text {unfaulted }}}{\dot{m}_{\text {unfaulted }}}$ \\
\hline
\end{tabular}

Table 5 shows the grid of fault conditions that were simulated with Cheung and Braun's [11] model, and compiled into input data set C.

Table 5: Fault conditions for input data set $\mathrm{C}$

\begin{tabular}{ll} 
Fault Type & Fault Intensity \\
\hline No fault & - \\
UC or OC & $70,80,90,110,120,130$ \\
EA & $90,75,60,45$ \\
CA & $90,77,63,50,40$
\end{tabular}




\begin{tabular}{ll} 
LL & $50,100,300,600,1200,2000,3500$ \\
NC & $10,30,45,80,100$ \\
VL & $10,20,35,50$ \\
\hline
\end{tabular}

When each of the 32 fault conditions is combined with the 48 operating conditions, it gives 1536 scenarios. When these 1536 scenarios are simulated for each of the 8 air conditioners, it gives 12,288 scenarios. However, the simulation uses an iterative solver that doesn't converge in some cases. In addition, the model is unable to simulate some conditions that are non-physical, such as very intense faults at certain operating conditions (in which a real air conditioner would likely trip a safety switch and shut down). These cases reduce the number of tests below 12,288 . Additional cases were generated in the proximity of conditions where the behavior of certain parameters, such as suction superheat, become nearly singular, for use in a different portion of this research. The resulting total number of scenarios in input data set $\mathrm{C}$ is 14,029 .

The final set, data set $\mathrm{D}$, is intended to better match the performance evaluation to expected deployment conditions. The weighting is applied as follows. We assume that the AFDD tool will be deployed in Omaha, Nebraska during times when ambient temperatures are above $60^{\circ} \mathrm{F}\left(15.6^{\circ} \mathrm{C}\right)$ that fall between $6 \mathrm{AM}$ and $7 \mathrm{PM}$. Typical meteorological year (TMY) weather data are used to calculate the probability of each outdoor temperature scenario. The distribution of faults is also weighted to an assumed fault distribution, which is described below. This distribution assumes that faults occur singly (i.e. multiple types of fault do not occur simultaneously), and probabilities are assigned for each fault type and for fault intensity (FI) bins within each fault type. These are assumed somewhat arbitrarily because there are no known reliable sources for actual fault distribution in the field.

The assumed distribution of fault type is shown in Table 6, using the nomenclature of Table 1. This distribution assumes that $39 \%$ of air-conditioners are fault free (NoF), meaning that they don't have a significant fault.

Table 6: Assumed distribution of fault types

\begin{tabular}{cc}
$\begin{array}{c}\text { Fault } \\
\text { Type }\end{array}$ & Probability \\
\hline NoF & $39 \%$ \\
UC & $10 \%$ \\
OC & $7 \%$ \\
EA & $13 \%$ \\
CA & $16 \%$ \\
LL & $5 \%$ \\
NC & $5 \%$ \\
VL & $4 \%$
\end{tabular}

When a given fault type occurs, it has some level of fault intensity (FI). The assumed distribution of FI for each fault type is shown in 
Table 7. This distribution gives the assumed incidence for each bin of FI. For example, if a UC fault occurs (based on the $10 \%$ probability in Table 6), then

Table 7 shows that there is a $28 \%$ probability that the FI $=80 \%$. Thus, the overall probability of a scenario with $80 \%$ charge is $2.8 \%(10 \% \times 28 \%=2.8 \%)$.

Table 7: Assumed fault intensity distribution by fault type

\begin{tabular}{|c|c|c|c|c|c|c|c|c|}
\hline $\begin{array}{c}\text { Fault } \\
\text { Type }\end{array}$ & FI & Incidence & $\begin{array}{c}\text { Fault } \\
\text { Type }\end{array}$ & FI & Incidence & $\begin{array}{l}\text { Fault } \\
\text { Type }\end{array}$ & FI & Incidence \\
\hline \multirow[t]{4}{*}{ UC } & $70 \%$ & $16 \%$ & CA & $40 \%$ & $5 \%$ & NC & $10 \%$ & $40 \%$ \\
\hline & $80 \%$ & $28 \%$ & & $50 \%$ & $11 \%$ & & $30 \%$ & $28 \%$ \\
\hline & $90 \%$ & $56 \%$ & & $63 \%$ & $19 \%$ & & $55 \%$ & $20 \%$ \\
\hline & & & & $77 \%$ & $27 \%$ & & $80 \%$ & $10 \%$ \\
\hline \multirow[t]{4}{*}{ OC } & $110 \%$ & $65 \%$ & & $90 \%$ & $38 \%$ & & $100 \%$ & $2 \%$ \\
\hline & $120 \%$ & $26 \%$ & & & & & & \\
\hline & $130 \%$ & $10 \%$ & LL & $50 \%$ & $31 \%$ & VL & $10 \%$ & $45 \%$ \\
\hline & & & & $100 \%$ & $24 \%$ & & $20 \%$ & $25 \%$ \\
\hline \multirow[t]{5}{*}{ EA } & $45 \%$ & $8 \%$ & & $300 \%$ & $16 \%$ & & $35 \%$ & $18 \%$ \\
\hline & $60 \%$ & $20 \%$ & & $600 \%$ & $12 \%$ & & $50 \%$ & $13 \%$ \\
\hline & $75 \%$ & $31 \%$ & & $1200 \%$ & $8 \%$ & & & \\
\hline & $90 \%$ & $41 \%$ & & $2000 \%$ & $6 \%$ & & & \\
\hline & & & & $3500 \%$ & $3 \%$ & & & \\
\hline
\end{tabular}

In the remainder of this paper, the effects of each set of inputs on the evaluation of three AFDD protocols will be compared and conclusions drawn. The AFDD protocols, identified as I, II, and III, use diverse approaches, have widely varying levels of complexity, and are each currently in widespread use. Each protocol appears to use a rulebased diagnosis approach. They range from very simple (AFDD I) to quite complex (AFDD III). AFDD I is a public-domain protocol specified in California's Title 24 energy code [20]. AFDD II and III were developed by private entities. Each of the protocols requires steady operating conditions to be deployed, and each specifies a range of operating conditions in which it can be applied. These protocols are believed to be representative of currently available AFDD protocols with similar deployment applicability.

\subsection{Results}

The results of applying the evaluation methodology described by [10] and [13] are presented for each of the four sets of inputs. Although a typical goal of application of AFDD performance evaluation is to compare and contrast the performance of one tool to the next, the purpose of the current paper is to show the effect of inputs on evaluation outcomes. Therefore, the plots in Figure 1 to Figure 11 each contain results from one protocol evaluated with all four sets of data individually.

In each plot, moving from set $\mathrm{A}$ to set $\mathrm{B}$ shows the difference between the laboratory measurement data and simulation data, because the simulation is conducted under the same conditions as the measurements were made at. Yuill et al. [14] point out that the differences don't necessarily suggest any shortcomings of the simulation. Measurements are subject to random error, unlike simulation, and the simulation is constrained to follow 
physical laws, such as mass and energy balances, so it is likely that the simulations give a more realistic scenario than the measurements in many cases.

The first result is the No Response rate. This is the percentage of cases in a data set for which the AFDD protocol is unable to give a response. This is normally because the operating conditions are outside the range for which the AFDD is intended to be applied. The results for each data set and each AFDD protocol are shown in Table 8.

Table 8: No Response rates for each protocol and each data set
\begin{tabular}{ccccc} 
Data Set & AFDD I & AFDD II & AFDD III \\
\hline A & $26 \%$ & $36 \%$ & $32 \%$ \\
B & $26 \%$ & $38 \%$ & $32 \%$ \\
C & $45 \%$ & $46 \%$ & $23 \%$ \\
D & $19 \%$ & $52 \%$ & $16 \%$
\end{tabular}

The results for set $\mathrm{C}$ are higher than $\mathrm{A}$ and $\mathrm{B}$, because this set includes all physical combinations of conditions shown in Table 3, many of which are at the extremes of normal operating conditions. Similarly, data set D has lower No Response rates for AFDD I and AFDD III because it contains inputs intended to match typical deployment conditions. AFDD II has high no response rates, generally, because in addition to typical operating range criteria, it also has criteria for certain variables in the refrigeration cycle, which appear to be quite restrictive. These are dependent variables, in the case of a model, and this explains why the No Response rates for AFDD II are different for Set A and Set B (which contain the same operating conditions).

\subsection{False Alarms}

Figure 1 to Figure 3 show the False Alarm results from evaluating the three protocols, following the methodology of [9-10] and [13] using each of the four data sets. The methodology defines a False Alarm as a scenario in which degradation of capacity or efficiency is less than a given threshold, but the AFDD protocol annunciates a fault. A fault may or may not be present, but if present it is not significant in its effect (significance being quantified with the chosen FIR threshold). For example, a user who feels that a loss of capacity or efficiency less than $5 \%$ is not significant would choose the results at the 95\% FIR threshold. 


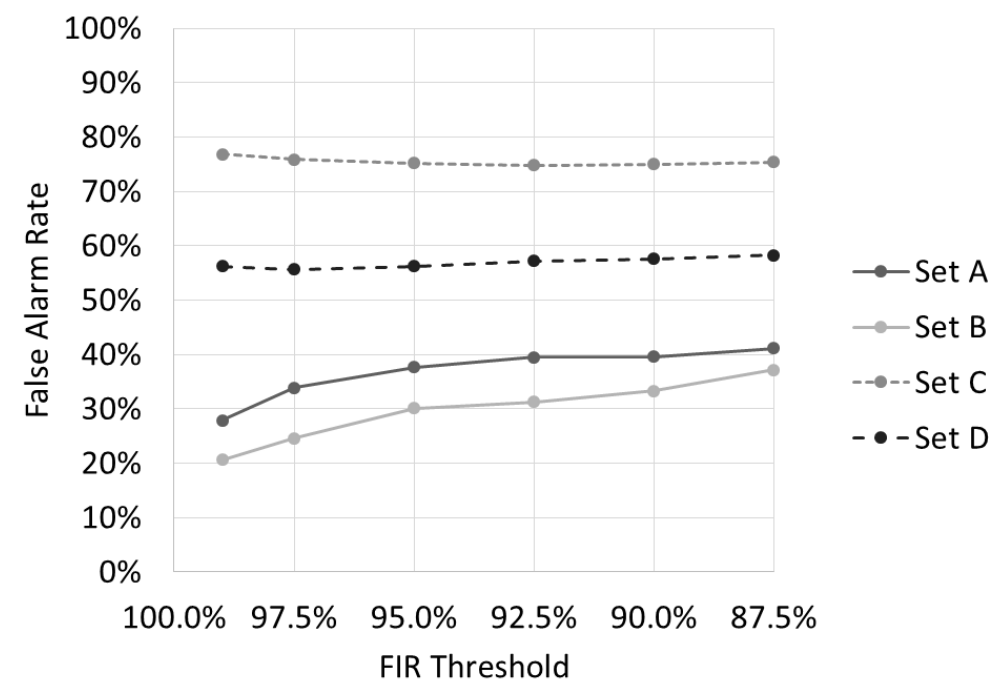

Figure 1: AFDD I False Alarm rates with four input sets

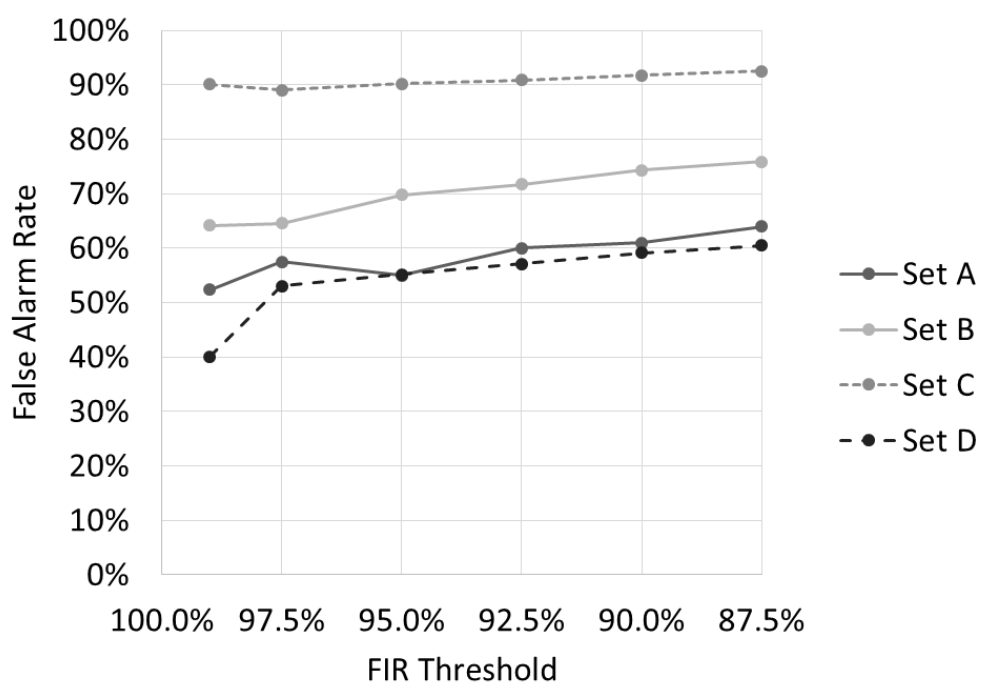

Figure 2: AFDD II False Alarm rates with four input sets 


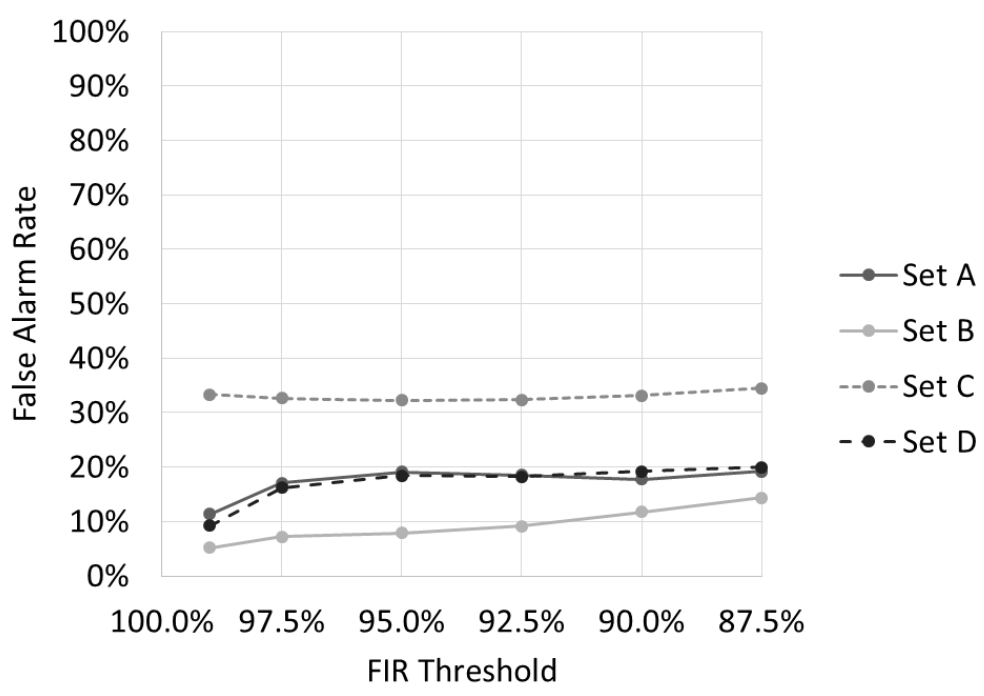

Figure 3: AFDD III False Alarm rates with four input sets

The results are higher than one might expect, particularly for AFDD I and AFDD II. They are also surprisingly flat, in many cases. We would expect the rate to increase as we move rightward (allowing larger and larger faults to be considered unfaulted). Overall, the faults severity seems not to affect the protocols' detection rates significantly.

Another surprising result is that for AFDD II and AFDD III, the results from Set A and Set $\mathrm{D}$ are nearly identical. This would suggest that the uncontrolled distribution from the measurement set happens to mimic quite well the distribution that is weighted according to the probability of occurrence during field deployment. However, by comparing the distributions in Table 1 and Table 6, we know that is not the case. We conclude that it is coincidental agreement. Particularly, since AFDD I does not exhibit the same close agreement.

A more expected result is that for all three AFDD protocols, the worst performance is with Set $C$. This is presumably because Set $C$ has so many scenarios at the more extreme conditions, as shown in Table 3. In particular, we have observed that many AFDD tools struggle with scenarios in which the evaporator coil is dry, which is likely to occur for the cases with indoor air at $12.8^{\circ} \mathrm{C}\left(55^{\circ} \mathrm{F}\right)$ wetbulb temperature.

We expect the results from Set A and Set B to agree, because they represent identical scenarios gathered with measurements and simulation respectively, but they don't agree particularly well. However, the differences are not significant compared with the differences from one protocol to the next, or even when compared with the range of differences from different input sets. Regardless of the confidence one has in the fidelity of the models, their ability to control conditions means that they are more effective for evaluating AFDD than the existing measurement set.

\subsection{Misdiagnoses}

Figure 4 to Figure 6 show the Misdiagnosis results from evaluating the three protocols. A Misdiagnosis is a case in which a fault is present and the AFDD correctly detects the presence of a fault, but it diagnoses a fault different from the actual fault. The results are 
grouped together in five bins of FIR. Although the methodology includes analysis on the basis of capacity and efficiency, only the efficiency results, Misdiagnosis ${ }_{\text {cop }}$, are presented in this paper, for the sake of brevity. In these figures, cases in which there weren't more than five scenarios in a bin are not presented, because they aren't considered meaningful. This typically only occurs for Set A and Set B, which are limited in number, and at the extremes of the FIR range. For example, in Figure 4, Set B has results only in the three middle FIR bins.

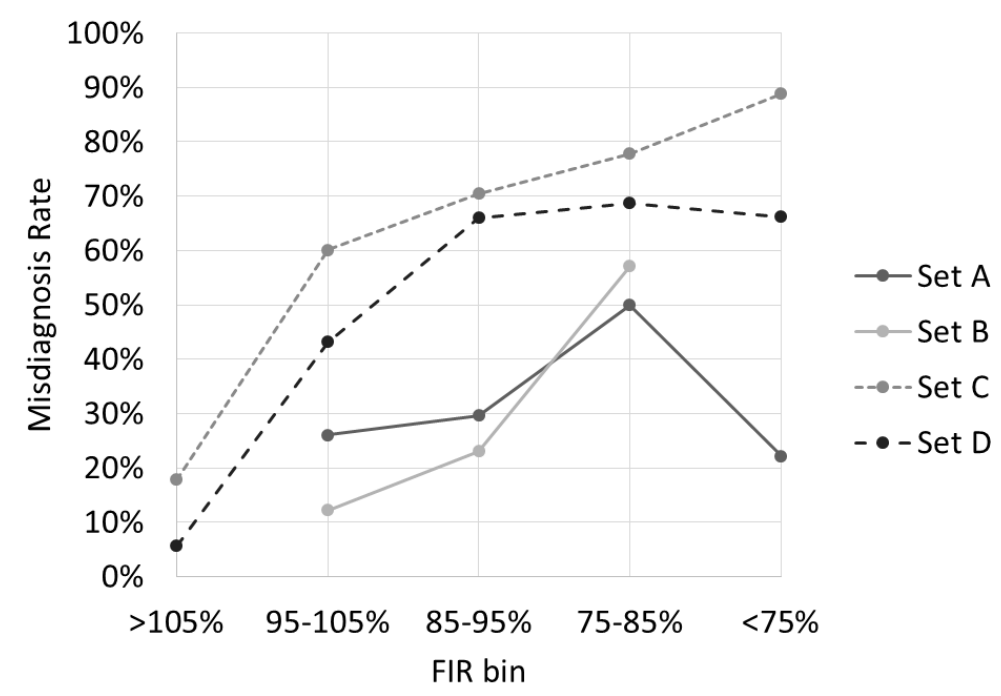

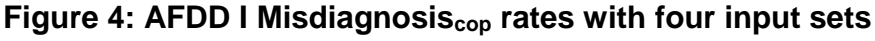

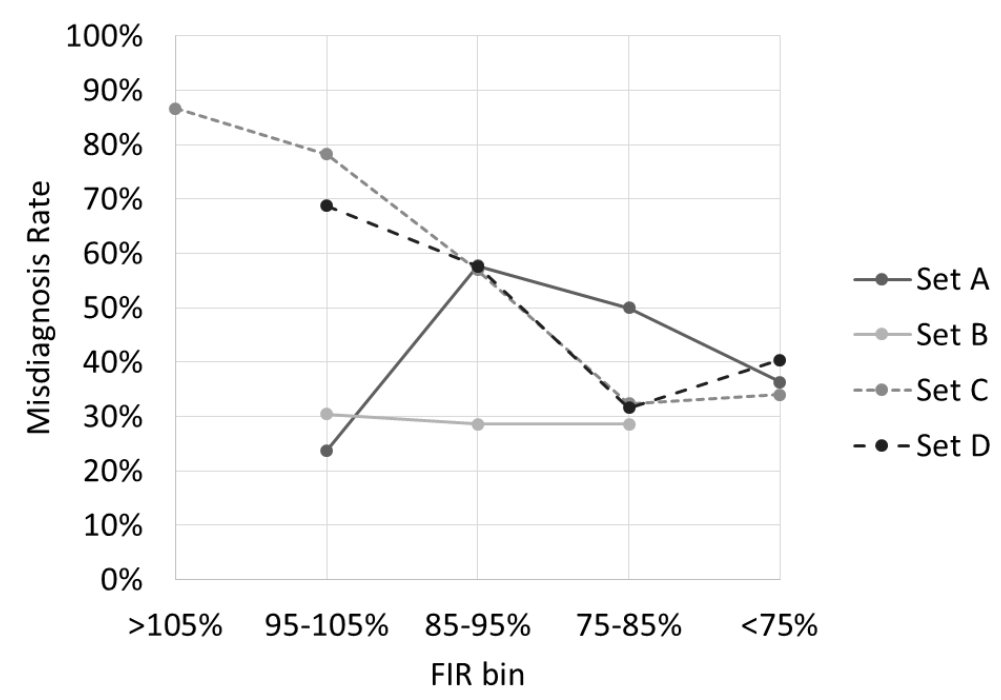

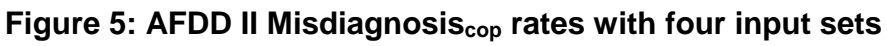




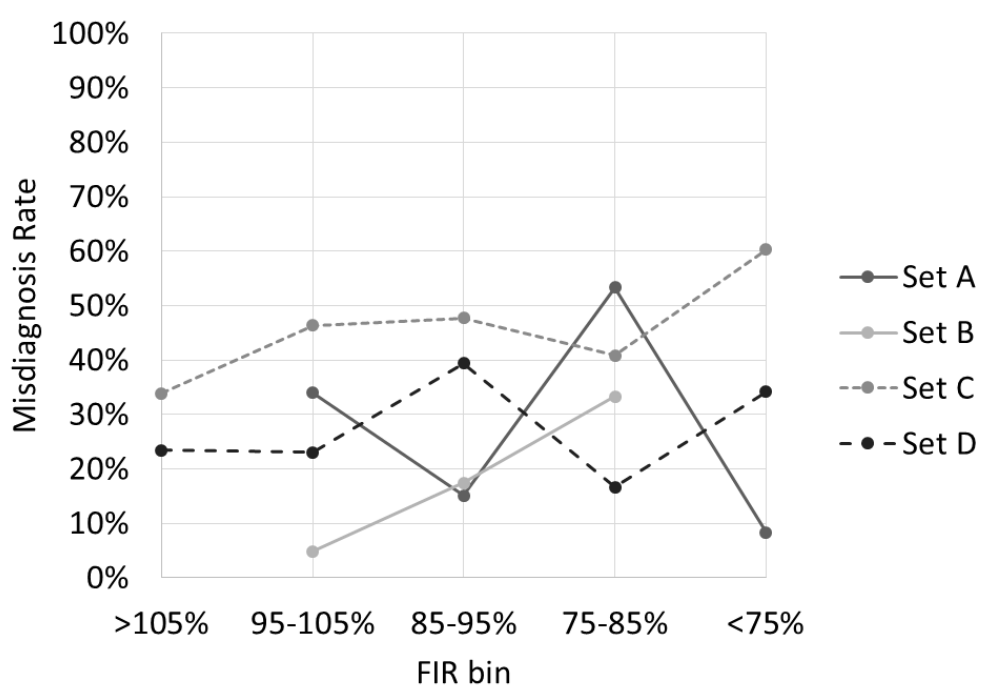

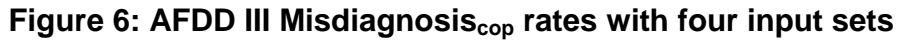

Misdiagnosis results tend to be more scattered than False Alarm results. This is partly because diagnosis algorithms typically are more sensitive to inputs than detection algorithms. It's also because a Misdiagnosis result is a blanket concept for all of the types of misdiagnosis that occur. For example, overcharge being diagnosed as an evaporator airflow fault; overcharge being diagnosed as a non-condensable gas fault; and so on. As with the False Alarms results, there is no consistent trend of better diagnosis for more severe faults.

AFDD I does not provide diagnoses for several of the fault types. It requires direct measurement of evaporator airflow (eliminating EA scenarios from the pool of potential Misdiagnoses), and provides diagnoses for charge faults only. Slightly overcharged systems can operate at higher efficiency under certain operating conditions. This may explain why the Misdiagnosis rates on the left of Figure 4 are lower; most of those cases are charge faults, which is the only diagnosis AFDD I can provide.

AFDD I performs significantly better (lower Misdiagnosis rate) with the conditions in the laboratory testing scenarios, whether measurement or simulation of measurements (Set A and Set B), than it does with the more evenly distributed conditions of Set C and Set D. This can be attributed to the heavy concentration of charge fault tests in the laboratory tests: 208 of the 480 tests with faults imposed, as shown in Table 2.

Each of the three protocols generally performs their diagnoses best with the simulated data at the laboratory conditions (Set B). This may be from the combined effect of (a) a large proportion of common fault and operation scenarios present in the laboratory set; and (b) the removal of measurement random error and non-physical values by the simulation.

Overall, the results in these figures are difficult to interpret, or to draw clear conclusions from. This suggests that additional methods or simplification of the results are required for more significant understanding of the performance of AFDD tools. 


\subsection{Missed Detections}

Figure 7 to Figure 9 show the Missed Detection results from evaluating the three protocols. A Missed Detection is a case in which a fault is present and the AFDD does not detect the presence of a fault. As with Misdiagnoses above, these results are grouped into FIR bins, and only the efficiency-related results are presented.

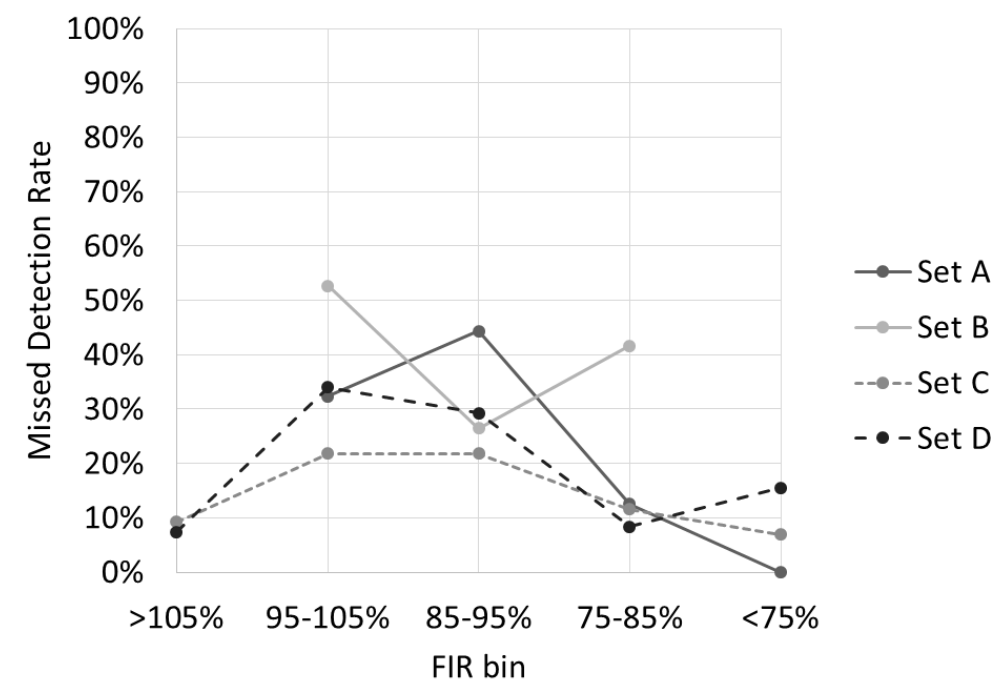

Figure 7: AFDD I Missed Detection ${ }_{\text {cop }}$ rates with four input sets

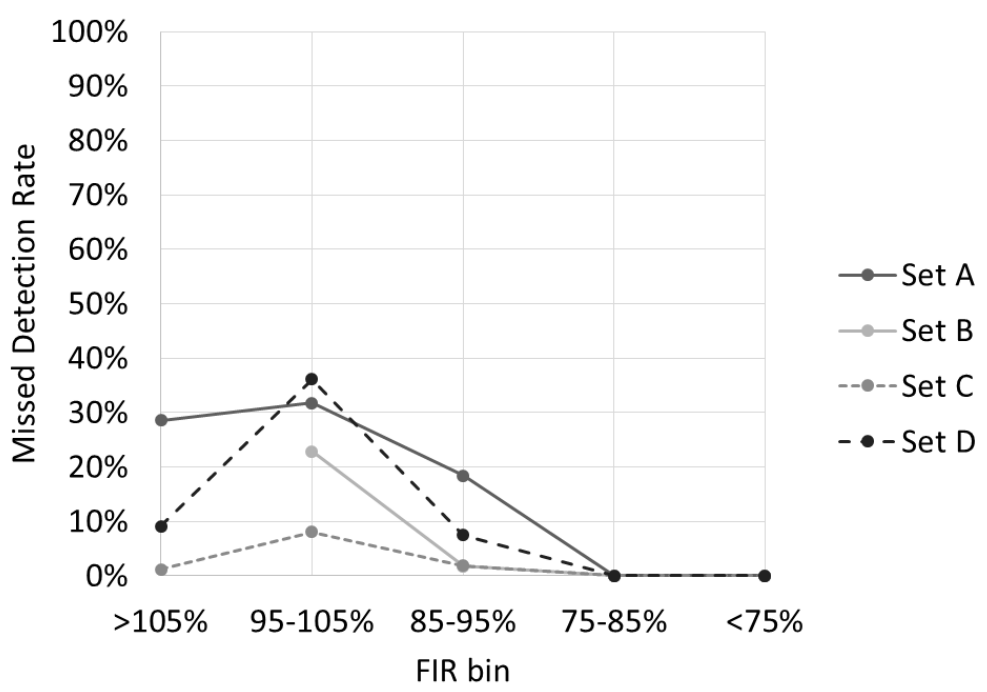

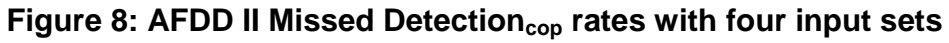




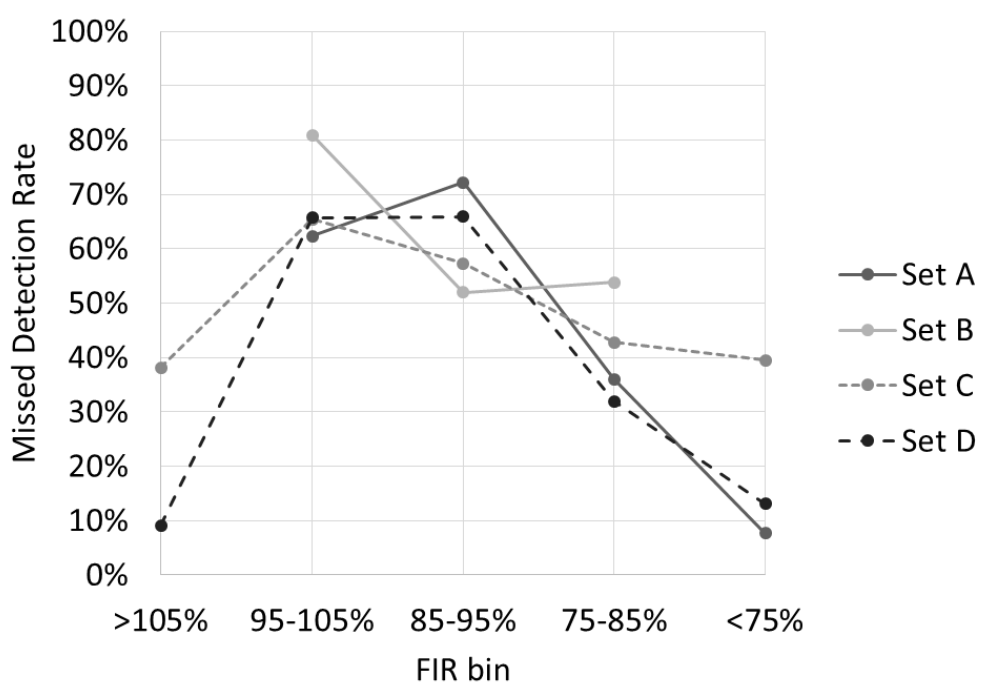

Figure 9: AFDD III Missed Detection ${ }_{\text {cop }}$ rates with four input sets

AFDD I and AFDD II had high False Alarm rates, as shown in Figure 1 and Figure 2. They have correspondingly low Missed Detection rates in Figure 7 and Figure 8. It could be argued that they are overly sensitive, because the cost of a False Alarm is typically greater than the cost of a Missed Detection.

AFDD III, in Figure 9, shows higher rates of Missed Detection than the other AFDD protocols. The developers of this protocol claim that it deliberately tolerates faults that are not cost-effective to address. In particular, faults that are not likely to reduce the life of the compressor significantly and faults that are very costly to address. This may explain why the Missed Detection rates are lower for the FIR > 105\% bin, which consists largely of overcharge faults. These can damage a compressor, but are relatively low-cost to address. Similarly, as faults become more severe (thus threatening the compressor, typically), on the right side of the plot, they are less frequently missed.

AFDD III doesn't perform as well with Set C (which includes an even distribution of fault conditions) at the higher fault severity levels. An examination of the individual outputs showed that this protocol almost never diagnoses liquid line restriction (LL) or compressor leakage (VL) faults. Each of these faults is prohibitively expensive to repair compared to their effect on operating cost, so it's reasonable to assume that LL and VL diagnoses are often diagnosed, but not annunciated by AFDD III. This could explain the increase in Missed Detections at the higher fault severity levels for Set C; this contains a higher proportion of LL and VL faults than the other sets.

Set B, which has a large proportion of charge faults, gets high Missed Detection rates from AFDD III. As discussed above, this protocol may be tolerating those faults when they're not too severe because repairing them often doesn't pay off, particularly for systems equipped with a thermostatic expansion valve (TXV).

\subsection{No Diagnosis}

Figure 10Figure 11 show the No Diagnosis results from evaluating two of the three protocols. A No Diagnosis case is one in which the AFDD correctly detects the presence of 
a fault, but does not provide a diagnosis for that fault. As with Misdiagnoses and Missed Detections above, these results are grouped into FIR bins, and only the efficiency-related results are presented. Since AFDD I does not give No Diagnosis responses (i.e. it always provides a diagnosis when it detects a fault), its No Diagnosis rate is $0 \%$ in all cases, so no corresponding plot for AFDD I is presented.

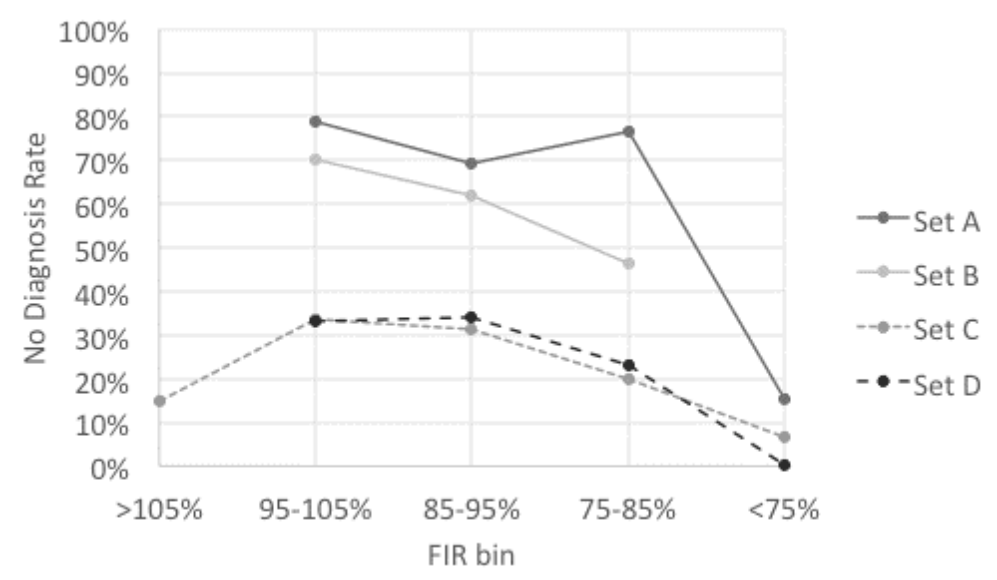

Figure 10: AFDD II No Diagnosis cop $_{\text {rates }}$ with four input sets

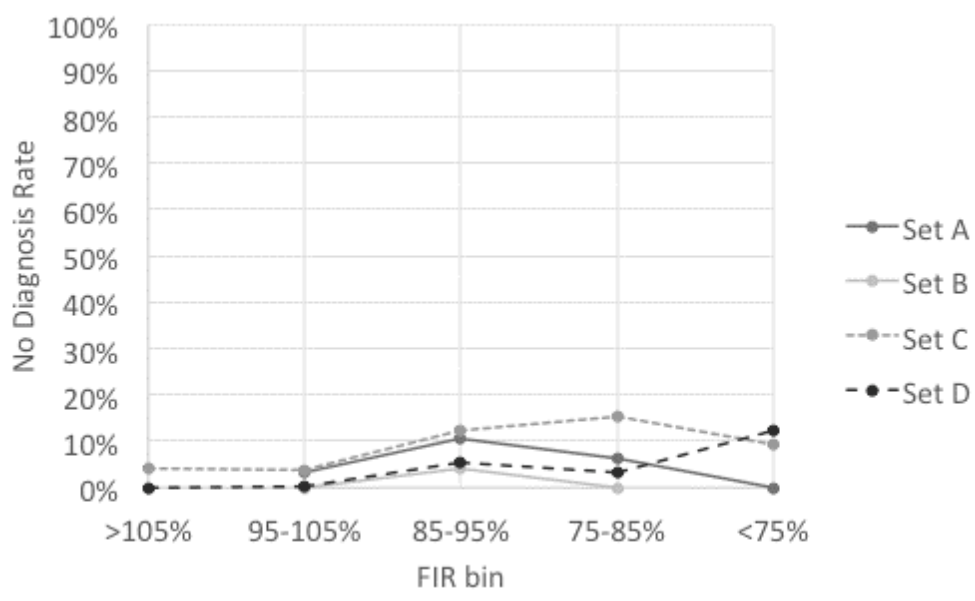

Figure 11: AFDD III No Diagnosis cop $_{\text {rates }}$ with four input sets

For AFDD II (Figure 10), there is a significant difference between the No Diagnosis rates at the laboratory experimental conditions (Sets A and B) and the rates at the controlled distribution conditions (Sets $\mathrm{C}$ and $\mathrm{D}$ ). The reasons for this are not obvious. It may be that the protocol is capable of detecting charge faults but has trouble diagnosing them (as noted above, the laboratory sets have a high proportion of charge faults).

Sets $\mathrm{C}$ and $\mathrm{D}$ (even distribution and weighted even distribution) give very similar results for AFDD II. We can conclude that this protocol's No Diagnosis rate is not sensitive to operating conditions, since the distribution of operating conditions for these sets is quite dissimilar, whereas the distribution of fault conditions is quite similar. 
AFDD III has quite low rates of No Diagnosis for each data set, with differences that are too small to be able to draw significant conclusions.

\subsection{Summary and Conclusions}

\subsection{Summary}

Three AFDD protocols have been evaluated using four different data sets. The evaluations show wide variations in performance. The performance doesn't follow predictable trends with respect to input conditions, but overall performance is significantly affected by input conditions.

\subsection{Conclusions}

\subsubsection{Performance of Currently Used Protocols}

- The lack of performance trends as a function of fault intensity shows that a comprehensive set of inputs is needed for meaningful evaluation.

- Potential adopters of an AFDD tool should consider the tool's performance, as evaluated in a meaningful and standardized fashion.

\subsubsection{Strategies for Effective Evaluation of AFDD Protocol Performance}

- Although some may be skeptical of models, the results show that model-induced uncertainty is very small compared to the effects of input conditions.

- The ability to produce a realistic input space, rather than a random or evenly distributed input space, supports simulation as the best method for providing AFDD evaluation input data

- These conclusions may be generalized across AFDD application areas (aerospace, nuclear, process control, etc.) if standard evaluation methods are proposed in those areas in the future.

\subsubsection{Future Work}

- It is not yet established what the distribution of conditions should be to represent realistic deployment scenarios.

- The results of the evaluation method are difficult to interpret. They should be tied to realistic deployment scenarios and represented in a simplified form. The authors have developed a single-number rating system that will be presented in a future paper to address this need.

\section{Acknowledgements}

The authors gratefully acknowledge support from the U.S. National Institute of Standards and Technology (NIST), New Buildings Institute, and California Energy Commission. We are grateful to Dr. Piotr Domanski of NIST for his technical insights in overseeing this project. 


\section{References}

[1] Li, H. and J.E. Braun. 2007. An overall performance index for characterizing the economic impact of faults in direct expansion cooling equipment. Int. J. of Refrigeration, 30 (2007): 299-310. doi:10.1016/j.ijrefrig.2006.07.026[2] Isermann, R. 1984. Process Fault Detection Based on Modeling and Estimation-A Survey. Automatica 20(4): 387-404. doi:10.1016/0005-1098(84)90098-0[3] Katipamula, S. and M.R. Brambley. 2005a. Methods for Fault Detection, Diagnostics, and Prognostics for Building Systems - A Review, Part I. HVAC\&R Research 11(1): 325. DOI:10.1080/10789669.2005.10391123

[4] Katipamula, S. and M.R. Brambley. 2005b. Methods for Fault Detection, Diagnostics, and Prognostics for Building Systems - A Review, Part II. HVAC\&R Research 11(2): 169-187. DOI:10.1080/10789669.2005.10391133

[5] Li, H. and J.E. Braun. 2009. Virtual Refrigerant Pressure Sensors for Use in Monitoring and Fault Diagnosis of Vapor-Compression Equipment. $H V A C \& R$ Research, 15 (3): 597-616. DOI:10.1080/10789669.2009.10390853

[6] Cho, J.M., J. Heo, W.V. Payne and P.A. Domanski. 2014. Normalized performance parameters for a residential heat pump in the cooling mode with single faults imposed. Applied Thermal Engineering 67(2014): 1-15. doi:10.1016/j.applthermaleng.2014.03.010

[7] Zhao, Y., F. Xiao, J. Wen, Y. Lu and S. Wang. 2014. A robust pattern recognitionbased fault detection and diagnosis (FDD) method for chillers. HVAC\&R Research 20(7): 798-809. DOI:10.1080/10789669.2014.938006

[8] Mulumba, T., A. Afshari, K. Yan, W. Shen and LK. Norford. 2015. Robust modelbased fault diagnosis for air handling units. Energy and Buildings 86(2015): 698707. doi:10.1016/j.enbuild.2014.10.069

[9] Yuill, D.P. and J.E. Braun. Evaluating Fault Detection and Diagnostics Protocols Applied to Air-cooled Vapor Compression Air-conditioners, Paper 2470, International Refrigeration and Air Conditioning Conference, (2012) July 16-19.

[10] Yuill, D.P. and J.E. Braun. 2013. Evaluating the performance of FDD protocols applied to air-cooled unitary air-conditioning equipment. HVAC\&R Research 19(7): 882-891. DOI:10.1080/10789669.2013.808135

[11] Cheung, H. and J.E. Braun. 2013a. Simulation of Fault Impacts for Vapor Compression Systems by Inverse Modeling Part I: Component Modeling and Validation, HVAC\&R Research 19(7): 892-906. DOI:10.1080/10789669.2013.824800

[12] Cheung, H. and J.E. Braun. 2013b. Simulation of Fault Impacts for Vapor Compression Systems by Inverse Modeling Part II: System Modeling and Validation, HVAC\&R Research 19(7): 907-921. DOI:10.1080/10789669.2013.819769 
[13] Yuill, D.P., H. Cheung and J.E. Braun. 2014b. Evaluation of Fault Detection and Diagnostics Tools by Simulation Results of Multiple Vapor Compression Systems. International Refrigeration and Air Conditioning Conference, Paper 2605, (2014) July 14-17.

[14] Yuill, D.P., H. Cheung and J.E. Braun. 2014a. Validation of a Fault-Modeling Equipped Vapor Compression System Model Using a Fault Detection and Diagnostics Evaluation Tool. International Refrigeration and Air Conditioning Conference, Paper 2606, (2014) July 14-17.

[15] Du, Z., P.A. Domanski and W.V. Payne. 2016. Effect of common faults on the performance of different types of vapor compression systems. Applied Thermal Engineering 98(2016): 61-72. doi:10.1016/j.applthermaleng.2015.11.108

[16] Zhou, Y., J. Liu and A.L. Dexter. 2014. Estimation of an incipient fault using an adaptive neurofuzzy sliding-mode observer. Energy and Buildings 77 (2014): 256269. doi:10.1016/j.enbuild.2014.02.001

[17] Frank, P. M. 1990. Fault diagnosis in dynamic systems using analytical and knowledge-based redundancy: A survey and some new results. Automatica, 26(3): 459-474. doi:10.1016/0005-1098(90)90018-D

[18] Braun, J. E. 2003. Automated fault detection and diagnostics for vapor compression cooling equipment. Journal of Solar Energy Engineering, 125(3): 266-274. doi:10.1115/1.1591001

[19] Armstrong, P. R., Laughman, C. R., Leeb, S. B., \& Norford, L. K. 2006. Detection of rooftop cooling unit faults based on electrical measurements. HVAC\&R Research, 12(1): 151-175. DOI:10.1080/10789669.2006.10391172

[20] California Energy Commission (CEC), 2012. 2013 Building Energy Efficiency Standards for residential and nonresidential buildings. CEC-400-2012-004-CMFREV2. Sacramento: California Energy Commission. http://www.energy.ca.gov/2012publications/CEC-400-2012-004/CEC-400-2012004-CMF-REV2.pdf 